\title{
SOME NEW RESULTS ON INFORMATION PERCOLATION
}

\author{
By Alain Bélanger and Gaston Giroux \\ Université de Sherbrooke
}

\begin{abstract}
We study the dynamics of a large number, $N$, of investors which have meetings by groups of $m$. We obtain an explicit formula for the solution of the associated non-linear system of differential equations. This formula is tractable and it enables us to show the asymptotic stability of a large class of models. One of those models can be thought of as an interacting portfolio market.
\end{abstract}

1. Introduction. Motivated by the study of dark markets, Duffie [2] introduces, in his research monograph, several models of Over-The-Counter ('OTC') markets. Chapter 3 (see also Duffie and Sun [8] and Duffie and Sun [9]) presents a mathematical foundation for the independent random matching of a large population. Here, we develop an approach inspired by Kac [13]. To do so, we start with a sequence of dynamical sets of interacting investors, one for each integer $N$.

We consider interactions involving $m$ investors, for a fixed $m \geq 2$, and we suppose that the intensities of these dynamics have an adequate dependence on $N$. Our techniques enable us to obtain an explicit formula for the associated non-linear system of differential equations. We thereby extend the results first obtained in Duffie and Manso [7] and pursued in Duffie, Giroux and Manso [4] (see also chapter 5 of Duffie [2]). We note that our formula is valid for any interaction kernel (for perfect and imperfect transmission of information) and it is more explicit than the one obtained for the particular kernel considered in these articles.

Our article is structured as follows. In section 2, we describe our dynamics, and then proceed to the statement and proof of our result. In section 3, we illustrate the tractability of our formula for a large class of models. In particular, we describe in some details one of the models of that class since it resolves a difficulty raised in Gârleanu [12], p. 547.

Received February 2012.

AMS 2000 subject classifications: 34A34, 82C31, 60G55.

Keywords and phrases: Large interacting sets, market equilibrium, Ordinary Differential Equations, continuous-time Markov chains. 
2. The dynamics. In order to build our model, we first need an integer, $N$, which represents the number of investors in the market. Secondly, we need another integer, $m$, with $2 \leq m \leq N$, which represents the fixed number of investors in each meeting. Thirdly, we need a symmetric probability kernel $Q$ on the product space $\left(E^{m}, \mathcal{E}^{\otimes m}\right)$ where $(E, \mathcal{E})$ is a measurable space. That is a function

$Q: E^{m} \times \mathcal{E}^{\otimes m} \rightarrow[0,1]$ such that:

(i) For each $\left(C_{1} \times \cdots \times C_{m}\right) \in \mathcal{E}^{\otimes m}$, the function $Q\left(x_{1}, x_{2}, \ldots x_{m} ; C_{1} \times\right.$ $\left.\cdots \times C_{m}\right)$ is measurable in $\left(x_{1}, x_{2}, \ldots x_{m}\right)$; and for each $\left(x_{1}, x_{2}, \ldots x_{m}\right)$ it is a probability measure in $\left(C_{1} \times \cdots \times C_{m}\right)$; and

(ii) For any permutation $\sigma$ of $\{1,2, \ldots, m\}$

$$
\begin{aligned}
& Q\left(x_{1}, x_{2}, \ldots x_{m} ; C_{1} \times \cdots \times C_{m}\right) \\
& \quad=Q\left(x_{\sigma(1)}, x_{\sigma(2)}, \ldots x_{\sigma(m)} ; C_{\sigma(1)} \times C_{\sigma(2)} \times \cdots \times C_{\sigma(m)}\right) .
\end{aligned}
$$

Lastly, we need a positive number, $\lambda$, representing the intensity of investor meetings. The higher $\lambda$ is, the lower the search frictions in the market.

With these objects we build a jump Markov process with values in $E^{N}$. Its state at time $t$ is denoted $\left(X_{1}^{N}(t), X_{2}^{N}(t), \ldots X_{N}^{N}(t)\right)$ and is thought of as the state of the market at time $t$. At each jump of a Poisson process with intensity $\lambda \frac{N}{m}$, a set of $m$ investors interacts according to the kernel $Q$. Groups are undistinguishable so each group has a probability of $\left(\begin{array}{c}N \\ m\end{array}\right)^{-1}$ of being involved in a given interaction. In doing so, we obtain a sequence of processes, one for each $N$. For simplicity, we now assume that $(E, \mathcal{E})=\left(\mathbb{R}^{d}, \mathcal{B}\left(\mathbb{R}^{d}\right)\right)$. In such a setting, we can deduce, from this sequence of processes, an associated system of ODE's using the same techniques as in Ferland and Giroux [11] (see also Bezandry et al. [1]). This implies that, for each time $t$, the laws of the sequence $\left(X_{1}^{N}(t)\right)_{N \geq m}$ converge to the probability law $\mu_{t}$ where $\mu_{t}$ is the solution of the Cauchy problem for the associated system of ODE's:

$$
\frac{d \mu_{t}}{d t}=\lambda\left(\mu_{t}^{\circ}-\mu_{t}\right) ; \quad \mu_{0}=\mu
$$

with

$$
\mu_{t}^{\circ m}(C)=\int_{\left(\mathbb{R}^{d}\right)^{m}} \mu_{t}\left(d x_{1}\right) \mu_{t}\left(d x_{2}\right) \ldots \mu_{t}\left(d x_{m}\right) \bar{Q}\left(x_{1}, x_{2}, \ldots x_{m} ; C\right)
$$

and

$$
\bar{Q}\left(x_{1}, x_{2}, \ldots x_{m} ; C\right)=Q\left(x_{1}, x_{2}, \ldots x_{m} ; C \times\left(\mathbb{R}^{d}\right)^{m-1}\right)
$$

for $C \in \mathcal{B}\left(\mathbb{R}^{d}\right)$. 


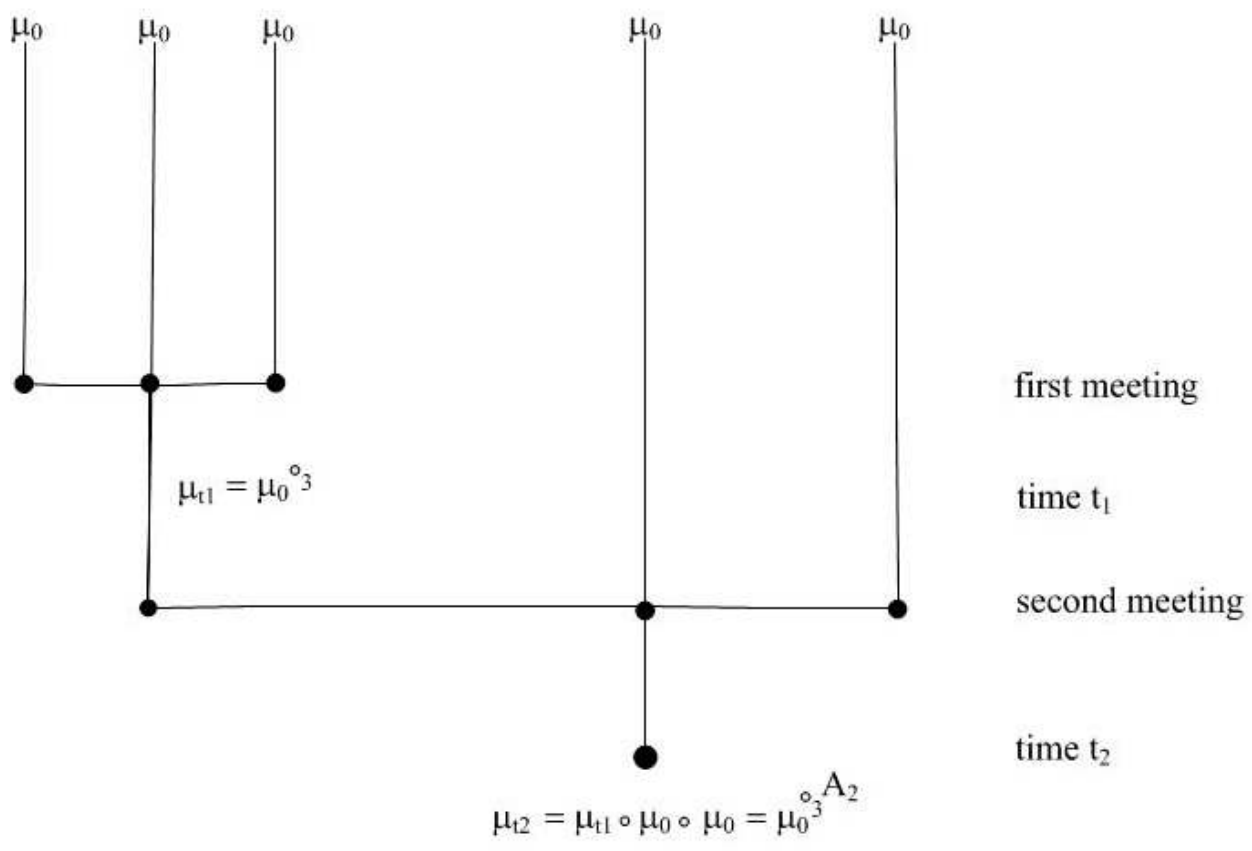

FIG 1. Simple interaction tree with only two meetings and $m=3$.

We can think of $\mu_{t}^{\circ}$ as the law at the root of the $m$-ary tree with only one interaction.

Here, we establish an explicit formula for the solution of the associated system of ODE's. In order to obtain the formula, we need to look at all the trees representing the interaction history of an investor up to time $t$. So for a tree, $A$, with at least one interaction, we divide the tree in $m$ subtrees at the node nearest to the root and continue recursively up to the leaves to define $\mu^{\circ}{ }_{m} A$. Figure 1 below gives a simple example of this operation. Let $\mathbb{A}_{n}$ be the set of all trees with $n$ interactions (a.k.a. nodes), each node producing $m$ branches. If $A_{n} \in \mathbb{A}_{n}$, then $\mu^{\circ m} A_{n}$ denotes the law obtained by iteration of $\mu^{\circ}$ through the successive nodes of the tree when we place the law $\mu$ on each leaf of $A_{n}$.

Hereafter the probability law defined as follows,

$$
\begin{aligned}
& \left(\nu_{1} \circ \nu_{2} \circ \cdots \circ \nu_{m}\right)(C) \\
& \triangleq \int_{E^{m}} \nu_{1}\left(d x_{1}\right) \nu_{2}\left(d x_{2}\right) \ldots \nu_{m}\left(d x_{m}\right) Q\left(x_{1}, x_{2}, \ldots x_{m} ; C \times E^{m-1}\right)
\end{aligned}
$$

will denote the law of an investor after the interaction of $m$ independent investors with law $\nu_{1}, \nu_{2}, \ldots, \nu_{m}$ respectively. 
Now we will show that our Cauchy problem has a unique solution which can be expressed, by conditioning on the number of interactions up to time $t$, and then by the investor's history. Such conditioning give us

$$
\mu_{t}=\sum_{n \geq 0} p_{n}(t) \frac{1}{\#_{m}(n)} \sum_{A_{n} \in \mathbb{A}_{n}} \mu^{\circ} A_{n}
$$

where $\#_{m}(n)=\prod_{k=1}^{n-1}((m-1) k+1)$ is the number of trees with $n$ nodes, taking into account their branching orders; and $p_{n}(t)=\frac{\#_{m}(n)}{(m-1)^{n} n !} e^{-\lambda t}(1-$ $\left.e^{-(m-1) \lambda t}\right)^{n}$ is the probability of having $n$ branchings up to time $t$. The uniqueness of the solution follows from a standard application of Grönwall's lemma (see Ferland and Giroux [11]).

Our main result is:

THEOREM 1. The convex combination,

$$
\mu_{t}=\sum_{n \geq 0} p_{n}(t) \frac{1}{\#_{m}(n)} \sum_{A_{n} \in \mathbb{A}_{n}} \mu^{\circ_{m} A_{n}}
$$

is the solution of the Cauchy problem

$$
\frac{d \mu_{t}}{d t}=\lambda\left(\mu_{t}^{\circ}-\mu_{t}\right) ; \quad \mu_{0}=\mu .
$$

Proof. Since the countable convex sum (1) is uniformly summable, we can differentiate $\mu_{t}$ term by term to obtain:

$$
-\lambda \mu_{t}+\lambda e^{-m \lambda t} \sum_{n \geq 1}\left(1-e^{-(m-1) \lambda t}\right)^{n-1} \frac{1}{(m-1)^{n-1}(n-1) !} \sum_{A_{n} \in \mathbb{A}_{n}} \mu^{\circ_{m} A_{n}}
$$

Thus we need to show that:

(2) $\mu_{t}^{\circ m}(C)=e^{-m \lambda t} \sum_{n \geq 0}\left(1-e^{-(m-1) \lambda t}\right)^{n} \frac{1}{(m-1)^{n} n !} \sum_{A_{n+1} \in \mathbb{A}_{n+1}} \mu^{\circ m} A_{n+1}(C)$

Starting with the definition (on page 2), we have that the LHS of (2) is equal to

$$
\begin{aligned}
\int_{E^{m}} & \left(\sum_{i_{1} \geq 0} e^{-\lambda t}\left(1-e^{-(m-1) \lambda t}\right)^{i_{1}} \frac{1}{(m-1)^{i_{1}} i_{1} !} \sum_{A_{i_{1}} \in \mathbb{A}_{i_{1}}} \mu^{\mathrm{o}_{m} A_{i_{1}}}\left(d x_{1}\right)\right) \ldots \\
\ldots & \left(\sum_{i_{m} \geq 0} e^{-\lambda t}\left(1-e^{-(m-1) \lambda t}\right)^{i_{m}} \frac{1}{(m-1)^{i_{m}} i_{m} !} \sum_{A_{i_{m}} \in \mathbb{A}_{i_{m}}} \mu^{\mathrm{o}_{m} A_{i_{m}}}\left(d x_{m}\right)\right) \ldots \\
& \ldots Q\left(x_{1}, \ldots, x_{m} ; C \times E^{m-1}\right)
\end{aligned}
$$


which is equal to

$$
\begin{aligned}
& \int_{E^{m}} e^{-m \lambda t}\left\{\sum_{n \geq 0}\left(1-e^{-(m-1) \lambda t}\right)^{n} \sum_{i_{1}+\cdots+i_{m}=n} \frac{1}{(m-1)^{n} i_{1} ! \ldots i_{m} !} \ldots\right. \\
& \left.\left(\sum_{A_{i_{1}} \in \mathbb{A}_{i_{1}}} \mu^{\circ_{m} A_{i_{1}}}\left(d x_{1}\right)\right) \ldots\left(\sum_{A_{i_{m}} \in \mathbb{A}_{i_{m}}} \mu^{\circ{ }^{\circ} A_{i_{m}}}\left(d x_{m}\right)\right)\right\} Q\left(x_{1}, \ldots, x_{m} ; C \times E^{m-1}\right)
\end{aligned}
$$

which in turn is equal to

$$
e^{-m \lambda t} \sum_{n \geq 0}\left(1-e^{-(m-1) \lambda t}\right)^{n} \frac{1}{(m-1)^{n} n !} F\left(i_{1}, \ldots, i_{m}, n, \mu, A_{i_{1}}, \ldots, A_{i_{m}}, Q, C\right)
$$

where

$$
\begin{aligned}
& F\left(i_{1}, \ldots, i_{m}, n, \mu, A_{i_{1}}, \ldots, A_{i_{m}}, Q, C\right) \\
& =\ldots \int_{E^{m}} \sum_{i_{1}+\cdots+i_{m}=n}\left(\begin{array}{c}
n \\
i_{1}
\end{array}\right)\left(\begin{array}{c}
n-i_{1} \\
i_{2}
\end{array}\right) \ldots\left(\begin{array}{c}
i_{m-1}+i_{m} \\
i_{m-1}
\end{array}\right)\left(\sum_{A_{i_{1}} \in \mathbb{A}_{i_{1}}} \mu^{\circ A_{m} A_{i_{1}}}\left(d x_{1}\right)\right) \ldots \\
& \ldots\left(\sum_{A_{i_{m}} \in \mathbb{A}_{i_{m}}} \mu^{\circ{ }_{m} A_{i_{m}}}\left(d x_{m}\right)\right) Q\left(x_{1}, \ldots, x_{m} ; C \times E^{m-1}\right)
\end{aligned}
$$

And this last expression is a decomposition of the trees $A_{n+1} \in \mathbb{A}_{n+1}$ appearing in the RHS of (2) in $m$ subtrees after the first node (taking the branching order into account). The two expressions are therefore equal and this proves the theorem.

REMARK 2. Our result brings a simplification to the special case studied in section 3 of Duffie, Giroux and Manso [4] where the term $a_{(m-1)(n-1)+1}$ can now be given explicitly as $\frac{1}{(m-1)^{n-1}(n-1) !}$. It also suggests that some of the results of Duffie, Malamud and Manso [6] can be extended to the case where information exchanges involve $m$ agents (with possibly $m$ random as they consider) even though the equation in their model does not belong to the class studied in the present paper and the techniques of tree expansions are not directly applicable. But a functional law of large numbers might be provable for their set of ODE's (4) and (5) in theorem 3.2 on page 1581.

REMARK 3. We call the law

$$
\mu_{t}=\sum_{n \geq 0} e^{-\lambda t}\left(1-e^{-(m-1) \lambda t}\right)^{n} \frac{1}{(m-1)^{n} n !} \sum_{A_{n} \in \mathbb{A}_{n}} \mu^{\circ_{m} A_{n}}
$$


an extended Wild sum, Wild [16], and note that the convex combination we obtain for the case $m=2$ is indeed the Wild sum,

$$
\mu_{t}=\sum_{n \geq 0} e^{-\lambda t}\left(1-e^{-\lambda t}\right)^{n} \frac{1}{n !} \sum_{A_{n} \in \mathbb{A}_{n}} \mu^{\circ_{m} A_{n}},
$$

well-known in the statistical physics of gases since the work of Kac [13].

3. Tractability. In Ferland and Giroux [10] the authors study a class of kinetic equations of Kac's type and they show, for binary collisions, a convergence to the steady state at an exponential rate. The convergence in Ferland and Giroux [10] is obtained along a set of convenient test functions with a telescoping technique due to Trotter [15] and with the use of a version of Wild sums obtained from judicious conditioning. We extend the results of Ferland and Giroux [10] for any $m \geq 2$.

Let $\mu$ denote a probability law on $\mathbb{R}$ and let $S_{m}(\mu)$ denote the law of $H_{1} X_{1}+H_{2} X_{2}+\cdots+H_{m} X_{m}$ where the random variables $\left\{X_{i}\right\}$ are independent and of law $\mu$ and the variables $\left\{H_{i}\right\}$ are independent of each other and of the $X$ variables. In terms of interaction kernels, we have $\bar{Q}\left(x_{1}, x_{2}, \ldots x_{m} ; C\right)=P\left[H_{1} x_{1}+H_{2} x_{2}+\cdots+H_{m} x_{m} \in C\right]$. Under very general conditions, Rösler [14] has shown that the transformation $S_{m}$ has a fixed point. In particular, if we suppose that the $H$-variables have values in $[0,1]$, that their mean is $\frac{1}{m}$ we then have $E\left[H_{1}+H_{2}+\cdots+H_{m}\right]=1$ and $S_{m}(\mu)$ has the same first moment as $\mu$. Moreover, if the $H$-variables are not Bernoulli we have that $E\left[H_{1}^{2}+H_{2}^{2}+\cdots+H_{m}^{2}\right]<1$ and then Rösler [14] gives us the existence of a fixed point, denoted $\gamma$, which is a steady state for the associated system of ODE's. This fixed point has a second moment as soon as $\mu$ does. Our goal is to establish the following result for the particular kernel $\bar{Q}$ just introduced.

THEOREM 4. If we suppose that $\mu$ has a finite second moment then we have that the law

$$
\mu_{t}=\sum_{n \geq 0} e^{-\lambda t}\left(1-e^{-(m-1) \lambda t}\right)^{n} \frac{1}{(m-1)^{n} n !} \sum_{A_{n} \in \mathbb{A}_{n}} \mu^{\circ m} A_{n}
$$

converges to $\gamma$ at the exponential rate $\eta=1-E\left[H_{1}^{2}+H_{2}^{2}+\cdots+H_{m}^{2}\right]$.

Our proof is an extension of the one in Ferland and Giroux [10] which treats the case $m=2$.

Proof. Let us first consider the tree with $m$ leaves, denoted $A_{1}$. On each one of its leaves put independent random variables of law $\mu$. Assume that 
these variables interact at a node to give $H_{1} X_{1}+H_{2} X_{2}+\cdots+H_{m} X_{m}$. Let us call $\mu^{\circ} A_{1}$, or more simply $\mu^{\circ}$, the law of this variable. In a similar fashion, we can consider $\gamma^{\circ}$ (which is $\gamma$ since it is a fixed point). We will first consider the differences $\left|\left\langle\mu^{\circ} m, f\right\rangle-\left\langle\gamma^{\circ_{m}}, f\right\rangle\right|$ for each $f \in C_{b}^{2}$. One way to bound this difference is to use the telescoping technique of Trotter [15] where we replace one by one (from the left say) the variables with law $\mu$ by variables with law $\gamma$. We then obtain a sum of $m$ terms of the form

$$
\left|<\gamma^{\circ k} \circ \mu^{\circ m-k}, f>-<\gamma^{\circ k+1} \circ \mu^{\circ m-k-1}, f>\right|
$$

which we will bound. In our particular models we can write down these expressions explicitly as

$$
\begin{aligned}
& \mid E\left[f\left(H_{1} Y_{1}+H_{2} Y_{2}+\cdots+H_{k} Y_{k}+H_{k+1} X_{k+1}+\cdots+H_{m} X_{m}\right)\right] \\
& \quad-E\left[f \left(H_{1} Y_{1}+H_{2} Y_{2}+\cdots+H_{k} Y_{k}+H_{k+1} Y_{k+1}+H_{k+2} X_{k+2}+\ldots\right.\right. \\
& \left.\left.\quad+H_{m} X_{m}\right)\right] \mid
\end{aligned}
$$

where $Y_{i}: i=1, \ldots k+1$ have law $\gamma$ and $X_{j}$, with $j=k+1, \ldots, m$, have law $\mu$. Let $R_{k}=H_{1} Y_{1}+H_{2} Y_{2}+\cdots+H_{k} Y_{k}+H_{k+2} X_{k+2}+\cdots+H_{m} X_{m}$. Then we have

$$
f\left(R_{k}+H_{k+1} X_{k+1}\right)=f\left(R_{k}\right)+f^{\prime}\left(R_{k}\right) H_{k+1} X_{k+1}+\frac{1}{2} f^{\prime \prime}\left(R_{k}^{*}\right)\left(H_{k+1} X_{k+1}\right)^{2}
$$

and $f\left(R_{k}+H_{k+1} Y_{k+1}\right)=f\left(R_{k}\right)+f^{\prime}\left(R_{k}\right) H_{k+1} Y_{k+1}+\frac{1}{2} f^{\prime \prime}\left(R_{k}^{* *}\right)\left(H_{k+1} Y_{k+1}\right)^{2}$.

Which in turn give us

$$
\left|<\mu^{\circ m}, f>-<\gamma^{\circ_{m}}, f>\right| \leq c E\left[\sum_{i=1}^{m} H_{i}^{2}\right] .
$$

We now need to iterate the process according to the different trees. Let $\widehat{A}_{n}$ denote the set of leaves of the tree $A_{n}$. Then the contribution of a leaf $u \in \widehat{A}_{n}$ through its interactions down to the bottom of the tree is a product of the variables $H_{i}$ with $i=1, \ldots, m$. Let us denote this product by $C_{u}$. For the tree $A_{n}$, the result of its interactions through the bottom of the tree will therefore go from $\sum_{u \in \widehat{A}_{n}} C_{u} X_{u}$, when all the variables put on leaves have law $\mu$, to $\sum_{u \in \widehat{A}_{n}} C_{u} Y_{u}$ when all the variables have law $\gamma$. Applying the same techniques as above, namely a (longer) telescoping and a Taylor series expansion, we get

$$
\left|<\mu^{\circ_{m} A_{n}}, f>-<\gamma^{\circ_{m}}, f>\right| \leq c E\left[\sum_{u \in \widehat{A}_{n}} C_{u}^{2}\right]
$$


Now let

$$
e_{n}=\frac{1}{(m-1)^{n} n !} \sum_{A_{n} \in \mathbb{A}_{n}} E\left[\sum_{u \in \widehat{A}_{n}} C_{u}^{2}\right] .
$$

We have that $e_{n} \leq c n^{a-1}$ where $a=\frac{1-\eta}{m-1}$ and $\eta$ is such that $1-\eta=$ $E\left[\sum_{i=1}^{m} H_{i}^{2}\right]$ by Lemma 5 below. Then proceeding as in Theorem 3 of Ferland and Giroux [10] with the extended Wild sum and replacing $1-\eta$ by $\frac{1-\eta}{m-1}$ we have that $\left|<\mu_{t}, f>-<\gamma, f>\right| \leq c e^{-\eta t}$ for all $f \in C_{b}^{2}$.

The remaining details of our proof are provided in the proof of the following lemma.

LEMMA 5. We have

$$
e_{n}=\left(\frac{1}{n}\right)\left(\frac{1-\eta}{m-1}\right)\left(1+e_{1}+\cdots+e_{n-1}\right)
$$

Thereforem $e_{n} \leq c n^{a-1}$ with $a=\frac{1-\eta}{m-1}$.

Proof. The decomposition of each tree $A_{n}$ in $m$ subtrees $\left\{A_{n}^{i}\right\}_{i=1}^{m}$ at the first node enables us to write $e_{n}$ as a sum of $m$ similar terms

$$
f_{i}=\frac{1}{(m-1)^{n} n !} \sum_{A_{n} \in \mathbb{A}_{n}} E\left[\sum_{u \in \widehat{A}_{n}} C_{u}^{2}\right]
$$

where $\widehat{A}_{n}^{i}$ is the set of leaves of the $i^{t h}$ subtree. It suffices to treat the case $i=1$. Let us decompose $\mathbb{A}_{n}$, the set of trees with $n$ nodes, by the number, $k$, of nodes of the subtree $A_{n}^{1}$. There are $\left(\begin{array}{c}n-1 \\ k\end{array}\right)(m-1)^{n-1-k}(n-1-k)$ ! such trees. Indeed, since we need to take into account the order of appearance of these nodes we have $\left(\begin{array}{c}n-1 \\ k\end{array}\right)$ choices for the appearances of $A_{n}^{1}$ 's nodes. Then we have $(m-1)^{n-1-k}$ ways to divide the remaining nodes in the other $m-1$ trees and finally, there are $(n-1-k)$ ! choices for the nodes' appearances. Note that this number simplifies to $\frac{(n-1) !}{k !}(m-1)^{n-1-k}$. If we denote by $\mathbb{A}_{n, k}$ the subset of $\mathbb{A}_{n}$ formed by the trees $A_{n}$ for which their subtree $A_{n}^{1}$ has $k$ nodes, we can then express $f_{i}$ as

$$
f_{i}=\frac{1}{(m-1)^{n} n !} \sum_{k=0}^{n-1} \sum_{A_{n} \in \mathbb{A}_{n, k}} E\left[\sum_{u \in \widehat{A}^{i}} C_{u}^{2}\right]
$$




$$
\begin{aligned}
& =\frac{E\left[H_{i}^{2}\right]}{(m-1) n} \sum_{k=0}^{n-1} \frac{1}{(m-1)^{k} k !} \sum_{A_{k} \in \mathbb{A}_{n, k}} E\left[\sum_{u \in \widehat{A}_{k}} C_{u}^{2}\right] \\
& =\frac{E\left[H_{i}^{2}\right]}{(m-1) n} \sum_{k=0}^{n-1} e_{k}, \text { with } e_{0}=1 .
\end{aligned}
$$

This proves the first assertion. A similar calculation to the proof of Lemma 3 in Ferland and Giroux [10] gives us the second result.

REMARK 6. The perfect information transmission kernel $\bar{Q}\left(x_{1}, x_{2}, \ldots x_{m}\right.$; $C)=\delta_{x_{1}+x_{2}+\cdots+x_{m}}(C)$ has been used as the basic interaction in many variations by Duffie and Manso [7], Duffie, Giroux and Manso [4], Duffie, Malamud and Manso [5]. One can wonder whether some of their results can be extended to more general kernels.

REMARK 7. One variant of the kernel $\bar{Q}\left(x_{1}, x_{2}, \ldots x_{m} ; C\right)=P\left[H_{1} x_{1}+\right.$ $H_{2} x_{2}+\cdots+H_{m} x_{m} \in C$ ] solves the difficulty raised in Gârleanu [12] on page 547 of extending his model to the non-linear dynamics of the DGP model, Duffie, Gârleanu and Pedersen [3]. Our result provides the existence and the stability of a steady state for such an extension. We only illustrate the situation where investors interact by pairs as in Gârleanu [12]. An interaction is the exchange of a random number of shares of a risky asset for cash. Denoting by $(X, Y)$ the random wealth (i.e. total cash holding) of a pair of investors, we suppose that the wealth $X$ becomes, after a transaction with an investor of wealth $Y, B(X+J Y)+(1-B)(1-I) X$. Where the random variables $B, J$ and $I$ are independent of each other and independent of the pair $(X, Y) ; B$ is a Bernoulli law of parameter $1 / 2 ; I$ and $J$ have the same law which is not a Bernoulli and they take their values in $[0,1]$. The interpretation of the transaction is the following. With probability $1 / 2$, the investor with wealth $X$ is a seller of the risky asset and then adds to his wealth a random fraction $J$ of the wealth $Y$ of the other investor. Also with probability $1 / 2$, this investor is a buyer who pays a fraction $I$ of his wealth to the investor with wealth $Y$. We can also write the wealth $X$ after interaction as $(1-(1-B) I) X+B J Y$ and then easily see that we are in the class of models we consider since $\bar{Q}(x, y ; C)=P[(1-(1-B) I) x+B J y \in C]$ $; E[(1-(1-B) I)+B J]=1$; and $\left.E[1-(1-B) I)^{2}+(B J)^{2}\right]<1$.

Acknowledgements. The first author would like to thank the Université de Sherbrooke and its Faculté d'administration for their startup grant. 


\section{REFERENCES}

[1] Bezandry, P. H., Ferland, R., Giroux, G. and Roberge, J. C. (1994). Une approche probabiliste de résolution d'équations non linéaires. In MeasuredValued Processes, Stochastic Partial Differential Equations and Interacting Systems (D. A. Dawson, ed.). CRM Proceedings 8 Lecture Notes 5 17-33. American Mathematical Society, Princeton, New Jersey. MR1278280

[2] Duffie, D. (2012). Dark Markets: Asset pricing and information transmission in over-the-counter markets. Princeton University Press.

[3] Duffie, D., GÂrleanu, N. and Pedersen, L. H. (2005). Over-the-Counter markets. Econometrica 73 1815-1847. MR2171326

[4] Duffie, D., Giroux, G. and Manso, G. (2010). Information percolation. American Economic Journal: Microeconomics 2 100-111.

[5] Duffie, D., Malamud, S. and Manso, G. (2009). Information percolation with equilibrium search dynamics. Econometrica 77 1513-1574. MR2561068

[6] Duffie, D., Malamud, S. and Manso, G. (2010). The relative contributions of private information sharing and public information releases to information aggregation. Journal of Economic Theory 145 1574-1601. MR2888913

[7] Duffie, D. and Manso, G. (2007). Information Percolation in Large Markets. American Economic Review 97 203-209.

[8] Duffie, D. and Sun, Y. (2007). Existence of Independent Random Matching. Annals of Applied Probability 17 386-419. MR2292591

[9] Duffie, D. and Sun, Y. (2012). The Exact Law of Large Numbers for Independent Random Matching. Journal of Economic Theory 147 1105-1139. MR2913254

[10] Ferland, R. and Giroux, G. (1991). An exponential Rate of Convergence for a Class of Boltzmann Processes. Stochastics and Stochastics Reports 35 79-91. MR1110992

[11] Ferland, R. and Giroux, G. (2008). Law of Large Numbers for Dynamic Bargaining Markets. Journal of Applied Probability 45 45-54. MR2409309

[12] GÂrleanu, N. (2009). Pricing and Portfolio Choice in Imperfect Markets. Journal of Economic Theory 144 532-564. MR2887303

[13] KAC, M. (1956). Foundations of kinetic theory. In Proceedings of the Third Berkeley Symposium on Mathematical Statistics and Probability III 171-197. University of California Press, Berkeley and Los Angeles, California. MR0084985

[14] Rösler, U. (1992). A fixed point theorem for distributions. Stochastic Processes and their Applications 42 195-214. MR1176497

[15] Trotter, H. (1959). An elementary proof of the central limit theorem. Archiv der Mathematik (Basel) 10 226-234. MR0108847

[16] Wild, E. (1951). On the Boltzmann equation in the kinetic theory of gases. Proceedings of the Cambridge Philosophical Society 47 602-609. MR0042999

Alain Bélanger

UNIVERSITY OF SHERBROOKE

FACUlty of Administration

2500, Université BLVD.

Sherbrooke, Canada, J1K 2R1

E-MAIL: alain.a.belanger@usherbrooke.ca
GASTON Giroux

410, Vimy Street, Apt. 1

Sherbrooke, Canada, J1J 3M9

E-MAIL: gasgiroux@hotmail.com 